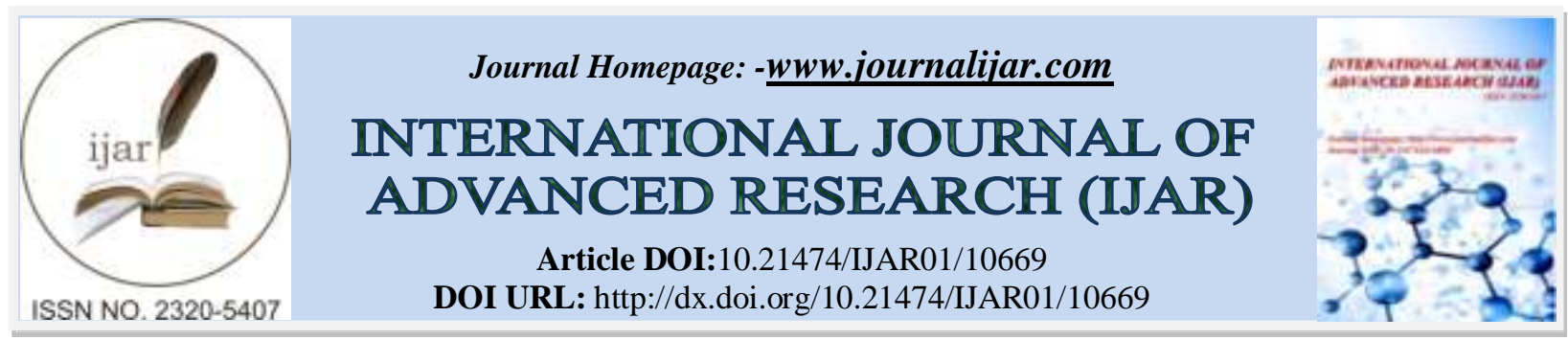

RESEARCH ARTICLE

\title{
COMPARATIVE EVALUATION OF MICROLEAKAGE OF DIFFERENT DENTIN REPLACEMENT MATERIALS
}

\section{Dr. Divya Chowdhary, Dr. Ramesh Chandra, Dr. Shailja Singh, Dr. Supratim Tripathi, Dr. Jyoti Jain and Dr. Urvashi Ojha}

\section{Manuscript Info \\ Manuscript History \\ Received: 15 January 2020 \\ Final Accepted: 17 February 2020 \\ Published: March 2020}

Key words:-

Microleakage, Stereomicroscope, Dentin

Replacement Material, Biodentine, MTA

\begin{abstract}
Microleakage can lead to staining around the margins of restorations, postoperative sensitivity, secondary caries, restoration failure, pulpal pathology or pulpal death, partial or total loss of restoration. Microleakage is usually associated with invasion from the external environment through the margins of the restoration, but microleakage can also occur internally.This study was carried out to evaluate in vitro the microleakage of four different dentin replacement materials ie.Group I - Glass inomer cement, Group II - Smart dentin replacement ,Group III - Biodentine,Group IV - MTA Angelus. On 60 samples class $\mathrm{V}$ cavity was prepared using a templete. Restoration was done following standard protocol. All samples were sectioned into two halves in buccolingual direction with a water-cooled slow speed diamond saw using air motor. Sample were viewed under Scanning Electron Microscope (100X) and stereomicroscope (40X).Photomicrographs were evaluated for both total length and adapted margin in $\mathrm{mm}$. All the materials considered in study showed gaps. But the highest marginal adaptability (\%) $95.70 \pm 1.29 \%$ and least microleakage $0.40 \pm 0.51$ was shown by Group II followed by Group III $80.13 \pm 5.67 \%$. So that we can say that the group which have maximum marginal adaptability, showed least microleakage and vice versa.
\end{abstract}

Copy Right, IJAR, 2020,. All rights reserved.

\section{Introduction:-}

In the current age of adhesive dentistry or microdentistry, conservation of tooth structure is paramount. Rather than using extension for prevention as a treatment guideline, emphasis now is placed on restriction with conviction ${ }^{4}$ In the era of esthetic dental adhesives, new materials and new techniques have been developed for tooth restoration, offering special handling characteristics and seeking to facilitate the daily practice of the clinician. ${ }^{8}$

One of the major objectives of tooth restoration is the protection of exposed dentine against bacteria and their toxins. The interface between the restoration and dental hard tissue is an area of clinical concern as insufficient sealing can result in marginal discoloration, secondary caries, and pulpitis. For that reason, adequate sealing is essential for optimal clinical performance. However, the literature is not always consistent with the terminology of leakage. Different levels of leakage are discussed, such as clearly detectable leakage, and not clinically detectable but with absence of secure adaptation. This 'hidden' leakage is usually denoted by the term microleakage. Microleakage may be defined as the clinically undetectable passage of bacteria, fluids, molecules or ions between a cavity wall and the 
restorative material. Clinically, microleakage can lead to staining around the margins of restorations, postoperative sensitivity, secondary caries, restoration failure, pulpal pathology or pulpal death, partial or total loss of restoration. Microleakage is usually associated with invasion from the external environment through the margins of the restoration, but microleakage can also occur internally. ${ }^{3}$

Regardless of the terminology used for leakage, the most desirable property that a restorative material should have is an adequate, complete and a lasting seal of the margins of the restoration.

Aim:

This study was carried out to evaluate in vitro the microleakage of four different dentin replacement materials.

\section{Materials \& Method:-}

A total 60 caries free human extracted molars were collected. The samples were cleaned to remove debris, calculus and rinsed with sodium hypochlorite to remove organic tissue and then stored in distilled water. Molars taken for the study were non-carious, non-restored, anomalous occlusal morphology was not included.

The 60 samples were taken out from the beaker and were dried and were divided into four groups of 15 each. Class $\mathrm{V}$ cavity preparation was done on all the teeth with ISO standardization no.245 bur (MANI) to standardize the cavities prepared in the extracted molars. The bur was replaced after preparation of every four cavities to maintain its efficiency of cutting. The dimension of the cavity was kept $2 \mathrm{~mm} * 3 \mathrm{~mm} * 8 \mathrm{~mm}$. All the cavities were standardized by preparing them by using a template prepared on graph paper. All the class V cavities were prepared with a high speed air-rotar under air water spray. After cavity preparation, the toilet of the cavity was performed and the cavity was dried.The distribution of groups was based on dentin replacement material and was as follows:

1. Group I - Glass inomer cement.(3M ESPE - Ketac ${ }^{\mathrm{TM}}$ Universal)

2. Group II - Smart dentin replacement (Dentsply)

3. Group III - Biodentine (Septodont)

4. Group IV - MTA Angelus (Angelus)

After restoration of cavities, finishing and polishing of the restorations was done in all samples. Samples were then stored in $100 \%$ humidity and placed in incubator at $37^{\circ} \mathrm{C}$ for $24 \mathrm{hrs}$. After incubation, all the tooth surfaces were covered with two coats of nail polish to within approximately $1 \mathrm{~mm}$ of the margin of the restoration. The specimens were immersed in $5 \%$ methylene blue at $37{ }^{\circ} \mathrm{C}$ for $48 \mathrm{hrs}$ and then rinsed with tap water. All samples were sectioned into two halves in buccolingual direction with a water-cooled slow speed diamond saw using air motor. All the samples were prepared by a single operator.

Sample were viewed under Scanning Electron Microscope (Zeiss -Evo18)at $100 \mathrm{X}$. Photomicrographs at 100X were taken. Scoring was done by two different evaluators using grids of $(1 \mathrm{~cm} \mathrm{x} 1 \mathrm{~cm})$. Therefore in order to simplify the calculations, these photomicrographs were evaluated for both total length and adapted margin in $\mathrm{mm}$.

Sample were viewed under Stereomicroscope (40X) observations recorded and statistically analysed.

Table 1:- Total and Adapted Length (mm) in different groups.

\begin{tabular}{|l|l|l|l|l|l|l|l|l|}
\hline Group & \multicolumn{3}{|l|}{ Group I } & \multicolumn{3}{l|}{ Group II } & \multicolumn{2}{l|}{ Group III } \\
\hline Sub No. & $\begin{array}{l}\text { TLM (in } \\
\text { mm) }\end{array}$ & $\begin{array}{l}\text { TAM (in } \\
\text { mm) }\end{array}$ & $\begin{array}{l}\text { TLM (in } \\
\text { mm) }\end{array}$ & $\begin{array}{l}\text { TAM (in } \\
\text { mm) }\end{array}$ & $\begin{array}{l}\text { TLM (in } \\
\text { mm) }\end{array}$ & $\begin{array}{l}\text { TAM (in } \\
\text { mm) }\end{array}$ & $\begin{array}{l}\text { TLM (in } \\
\text { mm) }\end{array}$ & $\begin{array}{l}\text { TAM (in } \\
\text { mm) }\end{array}$ \\
\hline 1 & 361 & 60 & 320 & 308 & 340 & 240 & 355 & 296 \\
\hline 2 & 352 & 59 & 431 & 407 & 421 & 349 & 368 & 253 \\
\hline 3 & 438 & 31 & 369 & 349 & 362 & 301 & 416 & 294 \\
\hline 4 & 372 & 42 & 364 & 355 & 398 & 335 & 374 & 236 \\
\hline 5 & 358 & 56 & 310 & 300 & 355 & 255 & 356 & 297 \\
\hline 6 & 350 & 55 & 433 & 410 & 425 & 353 & 363 & 248 \\
\hline 7 & 420 & 40 & 361 & 341 & 368 & 307 & 412 & 290 \\
\hline 8 & 380 & 39 & 358 & 349 & 401 & 338 & 377 & 239 \\
\hline 9 & 380 & 70 & 330 & 318 & 337 & 237 & 361 & 302 \\
\hline
\end{tabular}




\begin{tabular}{|l|l|l|l|l|l|l|l|l|}
\hline 10 & 365 & 64 & 435 & 411 & 419 & 346 & 363 & 248 \\
\hline 11 & 425 & 33 & 370 & 350 & 366 & 305 & 421 & 299 \\
\hline 12 & 378 & 44 & 371 & 362 & 391 & 328 & 373 & 235 \\
\hline 13 & 369 & 67 & 317 & 307 & 352 & 252 & 351 & 292 \\
\hline 14 & 340 & 51 & 444 & 421 & 429 & 357 & 366 & 251 \\
\hline 15 & 410 & 32 & 373 & 353 & 371 & 310 & 411 & 289 \\
\hline
\end{tabular}

As in Table 1 total length values in different groups ranged from 310 to $444 \mathrm{~mm}$ in different groups. The range was most confined in Group IV (351-421 mm) and most wide in Group II (310-444 mm). In Groups I and III the span of range was (340-438 mm and 337-429 mm). Adapted margin values ranged from 31 to $421 \mathrm{~mm}$. The range was most wide in Group II (300-421 mm) and most restricted in Group I (31-70 mm).

Table 2:- Intergroup comparison of marginal adaptability in different groups (ANOVA).

\begin{tabular}{|l|l|l|l|l|l|}
\hline Source of Variation & Sum of Squares & df & Mean Square & F & $\begin{array}{l}\text { p- } \\
\text { value }\end{array}$ \\
\hline Between Groups & 827078.067 & 3 & 275692.689 & 256.657 & $<0.001$ \\
\hline Within Groups & 60153.333 & 56 & 1074.167 & & \\
\hline Total & 887231.400 & 59 & & & \\
\hline
\end{tabular}

Analysis of variance shows a statistically significant intergroup difference $(\mathrm{p}<0.001)$. It was seen that marginal adaptation values were of higher order in Groups II and III and of lower order in Groups I and IV.Group II > Group III > Group IV > Group I

Table 3:- Microleakage values of different groups (mm).

\begin{tabular}{|c|c|c|c|c|c|c|c|c|}
\hline \multirow{2}{*}{$\begin{array}{l}\text { Sample } \\
\text { No. }\end{array}$} & \multicolumn{2}{|l|}{ Group I } & \multicolumn{2}{|l|}{ Group II } & \multicolumn{2}{|l|}{ Group III } & \multicolumn{2}{|l|}{ Group IV } \\
\hline & $\begin{array}{l}\text { Depth of } \\
\text { cavity }\end{array}$ & $\begin{array}{l}\text { Depth of } \\
\text { micro } \\
\text { leakage }\end{array}$ & $\begin{array}{l}\text { Depth of } \\
\text { cavity }\end{array}$ & $\begin{array}{l}\text { Depth of } \\
\text { micro } \\
\text { leakage }\end{array}$ & $\begin{array}{l}\text { Depth of } \\
\text { cavity }\end{array}$ & $\begin{array}{l}\text { Depth of } \\
\text { micro } \\
\text { leakage }\end{array}$ & $\begin{array}{l}\text { Depth of } \\
\text { cavity }\end{array}$ & $\begin{array}{l}\text { Depth of } \\
\text { micro } \\
\text { leakage }\end{array}$ \\
\hline 1 & 2.3 & 2.3 & 2.9 & 0 & 2.7 & 1.1 & 2 & 1.9 \\
\hline 2 & 2.3 & 2.3 & 2.8 & 0 & 2.8 & 1.2 & 2.6 & 1.8 \\
\hline 3 & 2.9 & 2.8 & 3 & 0.8 & 2.5 & 1.1 & 2.3 & 1.0 \\
\hline 4 & 2.3 & 2.3 & 2.5 & 0 & 2.7 & 1.2 & 2.5 & 2.4 \\
\hline 5 & 2.9 & 2.8 & 2.6 & 0 & 2.3 & 1 & 2.7 & 1.7 \\
\hline 6 & 2.7 & 2.7 & 2.5 & 0 & 2.6 & 0 & 2.8 & 1.9 \\
\hline 7 & 2.6 & 2.5 & 2.7 & 0.9 & 2.8 & 1.3 & 2.4 & 1.6 \\
\hline 8 & 2.4 & 1.7 & 2.7 & 0.6 & 2.5 & 1.2 & 2.6 & 1.1 \\
\hline 9 & 2.6 & 2.5 & 2.6 & 0 & 2.7 & 1.2 & 2.6 & 2.0 \\
\hline 10 & 2.3 & 2.3 & 2.5 & 0 & 2.4 & 1.8 & 2.5 & 1.8 \\
\hline 11 & 2.6 & 2.5 & 2.7 & 0.9 & 2.4 & 1.8 & 2.5 & 1.7 \\
\hline 12 & 2.7 & $\begin{array}{l}1.9 \\
\end{array}$ & 2.6 & 0 & 2.8 & 1.3 & 2.5 & 2.4 \\
\hline 13 & 2.5 & 2.4 & 2.9 & 0 & 2.5 & 1.7 & 2.6 & 2 \\
\hline 14 & 2.6 & 2.5 & 3 & 0.8 & 2 & 1.9 & 2.6 & 1.8 \\
\hline 15 & 2.7 & 2.7 & 2.8 & 0.8 & 2.7 & 1.7 & 2.4 & 1.6 \\
\hline
\end{tabular}

On intergroup comparison of microleakage using Kruskal-Wallis test, a significant difference was observed among groups. Values in Groups I were of higher order whereas values in Groups III and IV were of middle order \&Group II had values of lower order.Comparison using Mann-Whitney U test revealed statistically significant differences for all the comparisons. On the basis of above evaluations, the following order of microleakage was observed: Group I > Group IV > Group III > Group II

On the basis of \% Adapted Margin, the quality of Dentin Replacement Material can be categorized into four groups 1) \% adapted margin 0-25\% -Poor 2)25-50\%- Fair 3) 50-75\% -Good 4) 75-100\%-very good. Based on this criterion there was highly significant $(\mathrm{p}<0.001)$ difference between the qualities of the four dentin replacement materials. The best one was found to be Smart Dentin Replacement (100\% at very good quality) followed by 
Biodentine $(73.3 \%$ very good \& $26.7 \%$ good). The poorest dentin replacement material was found to be Glass Inomer Cement (100\% poor).

\section{Discussion:-}

Microleakage is an important property that has been used in assessing the success of any restorative material used in restoring a tooth. ${ }^{5} \mathrm{~A}$ major objectives of tooth restoration is to protect exposed dentine against bacteria and their toxins. The interface between the restoration and dental hard tissue is an area of concern as insufficient sealing can lead to marginal discoloration, secondary caries, and pulpitis. Thus, adequate sealing is essential for optimal clinical performance. Microleakage is truly a quantitative method that assesses the entire circumference of the toothrestoration interface and it is non-destructive, allowing marginal qualities to be assessed before and after exposure of the specimens to thermocycling. ${ }^{7}$

Detection of microleakage is accomplished with a number of techniques, including bacteria, chemical or radioactive tracer molecules, fluid permeability, and dye penetration. ${ }^{1}$ The most common technique being the use of dyes penetration. The immersion times of the specimens in the dye, which range from $1 \mathrm{hr}$ to 2 weeks in several studies which seem to have no influence on the microleakage results. ${ }^{6}$ There are different types of dyes that can be used for this purpose like Rhodamine-b dye, methylene blue, saffron,etc. but the most commonly preferred is methylene blue as it is a handy, colorful compound that can be easily detected under the microscope. Therefore the dye leakage method was performed in the present study to evaluate the microleakage and the sectioned samples were observed under stereomicroscope at 40X magnification to evaluate the amount of microleakage that has occurred in different groups.The origin of marginal leakage is very complex because of the numerous factors like polymerization of composite resins that produces shrinkage and subsequent water sorption that produces expansions; and also other factors like coefficient of thermal expansion also contribute to the quality of marginal adaptation as well. ${ }^{2}$

Marginal adaptation is defined as the interfacial distance between the restoration and the tooth structure. Excellent marginal adaptation depends on the quality of the restorations and adhesive systems. Marginal cavity adaptation of restorations in bonded dentin cavities reflects complex interactions between adhesive bonding on the one hand, and polymerization contraction strain, stress and elastic modulus, on the other. ${ }^{2}$

Within the limitations of laboratory studies, quantitative marginal analysis by SEM has proven to be an exact and reliable assessment method for the evaluation of the marginal adaptation of adhesive restorations. ${ }^{7}$ The use of SEM provides a means of visual observation of the adaptation of restoration to cavity walls because of its high magnification and depth of focus and therefore, this method was preferred in the present study to evaluate the marginal adaptability of different restorative materials.

The materials chosen in this study were glass ionomer cement, Smart dentin replacement, Biodentine and MTAAngelus; as all of them are dentin replacement materials .After applying the dentin replacement material, It was found that percentage margin adaptation comes out to be best in case of smart dentin replacement material (95.70\%) followed by Biodentine (80.13\%). The least percentage margin adaptation was found in Glass Inomer Cement $(13.28 \%)$. The result of the present study revealed that SDR showed the least microleakage followed by Biodentine and MTA when compared with GIC base.

The inability of GIC to produce an effective seal can be attributed to two factors: The material's sensitivity to moisture during placement and the dehydration after setting resulting in crazing and cracking. Glass ionomer sets as a result of a reaction between an acid and a base, with the product of the reaction, a hydrogel salt, acting as a binding matrix. GIC has properties like the ability to adsorb permanently to hydrophilic surfaces of hard oral tissues, capacity of fluoride ion release and uptake. However, in the cavity or in areas where proper isolation cannot be achieved glass ionomer cannot be used, as moisture and contaminants not only influence the bond strength of glass ionomer tooth; but negatively influence the physicomechanical properties of materials as well.

To overcome these disadvantages of GIC various materials like MTA and Biodentine ${ }^{\mathrm{TM}}$ were introduced. MTA shares many properties with glass ionomer as it can form chemical bond with tooth structure, compressive strength is comparable to dentin, high sealing ability, high biocompatiblity and radioopacity. ${ }^{9}$

The advantage of MTA over to glass ionomer is that it is not sensitive to moisture; in fact, moisture is necessary for its setting reaction. The sealing ability of MTA is not influenced by contamination with blood. Despite its good 
physical, biological properties and being hydrophilic in nature, its disadvantages include that the material is expensive, difficult to handle and long setting time. Hence, the search for an alternative to MTA material is aimed to reduce cost, setting time, improve handling properties, biocompatibility, and to increase feasibility to both professional and patient.

In the present study, Biodentine showed significantly less microleakage than MTA and GIC. It can be attributed to the fact that Biodentine is composed of a mixture of $5 \mu \mathrm{m}$ round particles embedded in a calcium silicate hydrate matrix which are much smaller than the size of MTA particle. Raskin et $\mathbf{a l}^{\mathbf{1 0}}$ investigated the microleakage of Class II sandwich technique with a biocompatible calcium silicate based dentin substitute and found that Biodentine can be indicated in open sandwich Class II restorations without any preliminary treatment.

Biodentine proves superior to MTA as it does not require a two step placement and the setting is faster, so there is a lower risk of bacterial contamination?.

When Biodentine come in contact with dentin, it results in the formation of tag like structures along the side of an interfacial layer. This is called as "mineral infiltration zone," where the alkaline caustic effect of the calcium silicate cement's hydration products degrades the collagenous component of the interfacial dentin.

For SDR, it could be attributed to the fact that SDR material that has very low polymerization shrinkage coupled with exceptionally low polymerization stress. In addition, SDR material possesses a high depth of cure and degree of conversion that optimizes physical and mechanical properties.

It is the first flowable composite base suitable for placement in cavities which can result in time savings of up to $30 \%$ over traditional while unique in its polymerization mechanism, remains compatible with all methacrylate based chemistries, including total or self-etch adhesives and universal composites restoratives. The optimized handling and self-leveling consistency of the SDR material is ideal cavity adaptation, especially in posterior restorations. Finally, the universal shade of SDR material enables simplified placement options.

Although all materials used in the study can be used as a suitable base (dentin replacement material),SDR exhibits superior marginal sealing ability as well as marginal adaptation and confirms best as dentin substitute compared to Biodentine, MTA and GIC.

\section{Conclusion:-}

The results of the study showed that all the materials considered in our study showed gaps. But the highest marginal adaptability (\%) $95.70 \pm 1.29 \%$ and least microleakage $0.40 \pm 0.51$ was shown by Group II followed by Group III $80.13 \pm 5.67 \%$. So that we can say that the group which have maximum marginal adaptability, showed least microleakage and vice versa.

\section{The order of marginal adaptability obtained was:} Group II > Group III > Group IV > Group I

\section{The order of microleakage obtained was:} Group I > Group IV > Group III > Group II

\section{Bibliography:-}

1. Alani AH and Toh CG. (1997):Detection of microleakage around dental restorations: a review. Operative Dentistry, 22:173-185.

2. El-Nawawy, M. Koraitim L, Abouelatta O,Hanan Hegazi. (2012):Marginal Adaptation of Nanofilled, Packable and Hybrid Dental Composite Resins Stored in Artificial Saliva. J. Biomed Eng., 2:105-114.

3. Fabianelli A, Pollington S, Davidson CL, Cagidiaco MC, Goracci C. (2007): The relevance of microleakage studies. Int Dent SA.. Jun;9(3):64-74. [Google Scholar].

4. Gupta KV, Verma P and Trivedi A.(2011): Evaluation of microleakage of various restorative materials: An in vitro study. Life Sci. J.;3:29-33. 
5. Gupta SK, Gupta J, Saraswathi V., BallalVasudev, Acharya Shashi Rashmi,(2012): Comparative evaluation of microleakage in Class $\mathrm{V}$ cavities using various glass ionomer cements: An in vitro study. J. Interdiscip. Dentistry,2:164-169.

6. Hilton TJ. (1998):Can modern restorative procedures and materials reliably seal cavities? In vitro investigations. Transactions Academy of Dental Materials,12:21-71.

7. Krejci I and Lutz F.(1991): Marginal adaptation of class V restorations using different restorative techniques. J. Dent.19:24-32.

8. Mali P., Deshpande S., and Singh A. (2006):Microleakage of restorative materials: An in vitro study. J. Indian Soc. Pedod. Prev. Dent.22:154-159.

9. Niranjan B, Shashikiran ND, Singla S, Thakur R, Dubey A, Maran S. (2016):A comparative microleakage evaluation of three different base materials in Class I cavity in deciduous molars in sandwich technique using dye penetration and dentin surface interface by scanning electron microscope. J Indian SocPedodPrev Dent;34:324- 30

10. Raskin A, D Hoore W, Gonthier S,Degrange M, Déjou J.(2001); Reliability of in vitro microleakage tests: A literature review. J. Adhes. Dent.,;3:295-308. 